\title{
Prenatal diagnosis of congenital heart disease in the northern region of England: benefits of a training programme for obstetric ultrasonographers
}

\author{
S Hunter, A Heads, J Wyllie, S Robson
}

\begin{abstract}
Objective-To examine the results of fetal cardiac scanning and audit the changes in performance resulting from the introduction of a training programme for obstetric ultrasonographers. Methods-Using the database of the Northern Regional Congenital Abnormality Survey (NORCAS), fetuses with complex or significant congenital heart disease (CCHD) diagnosed prenatally in 1994 were identified. A simple programme of centralised and local training was instituted in 1995 by the department of paediatric cardiology to teach obstetric ultrasonographers in district general hospital maternity departments to identify congenital heart malformations. The results of the training programme were assessed by comparing the 1994 identification rate of CCHD with the rates for 1996 and 1997.

Results-Birth rate fell during the study from 35026 in 1994 to 32874 in 1997. Registration of CCHD also fell, from 115 in 1994 to 87 in 1997. Prenatal recognition of CCHD rose from $17 \%$ in 1994 to $30 \%$ in 1995 and $36 \%$ in 1996 . In 1997 it fell slightly to $26.9 \%$. The total number of scans did not change much year on year, but the number of parents choosing termination increased significantly (from $22.7 \%$ to $57 \%$ ).

Conclusions-A simple training programme for obstetric ultrasonographers increased their ability to detect serious congenital heart disease at a routine 18-20 week anomaly scan. With a termination rate of more than $50 \%$, the incidence of CCHD in the population fell from 3.3/1000 to $2.6 / 1000$ live births. This audit, conducted within a stable population using ascertainment by a well established fetal malformation registry, suggests that prenatal diagnosis may have a significant effect on the incidence of complex or serious congenital cardiac malformations. (Heart 2000;84:294-298)
\end{abstract}

Keywords: congenital heart disease; fetal echocardiography

Fetal echocardiography and antenatal detection of congenital heart disease has been available in the northern region of England for more than a decade. Initially fetal cardiac scans were performed either because of maternal disease such as diabetes or a positive family history of congenital heart disease. As the service developed, contact with paediatric cardiologists in some district general hospitals resulted in obstetric scanners learning to recognise the four chambered view of the fetal heart.

For fetal cardiology to make a clinical impact, scanning of the fetal heart had to be extended to all the fetuses undergoing anomaly scanning (low risk pregnancies). The most skilful cardiac scanners work in paediatric cardiology units. It was obviously impossible for such individuals to scan all fetuses during routine anomaly scanning. A suggested alternative was to teach obstetric ultrasonographers to scan the fetal heart. ${ }^{12}$ The potential of such a programme had been described from a tertiary referral unit (Guy's Hospital, London) serving many district general hospitals. ${ }^{3}$ However, the true value of such a teaching initiative could only be demonstrated by a proper population study based on well documented clinical data.

The northern region has a stable population of 3.1 million, a birth rate of around 35000 at the outset of the study, and well defined borders. By the early 1990s every maternity unit was offering an anomaly scan at 18-20 weeks, and more than $96 \%$ of women in the region were having ultrasound screening. ${ }^{4}$

A survey of the prenatal cardiac activity in this period suggested that the existing service was not as effective as it might be, and the ultrasonographers were identifying only $18 \%$ of major cardiac malformations. ${ }^{4}$ It was therefore decided to set up a regional training programme aimed at incorporating cardiac scanning into the routine obstetric scanning practice. In this paper we report the methods that we employed, assess the effectiveness of the training, and examine the impact on congenital heart disease in the population of the region.

\section{Methods}

The cooperation of all 16 obstetric units in the region was sought, along with permission to visit each unit to explain the project. The initiative was generally welcomed and eventually we enjoyed complete cooperation from all maternity units.

The first visit was arranged to allow maximum attendance of local clinicians and radiographers. Two of us ( $\mathrm{SH}$ and $\mathrm{AH}$ ) spent a full day in the local unit. There was an illustrated lecture indicating the sorts of clinical problems that patients with congenital heart disease encounter and the sorts of treatment available at the regional centre for paediatric cardiology. The types of malformation likely to 
Table 1 Significance classification system currently in use in the paediatric cardiology database

\begin{tabular}{|c|c|}
\hline$C$ & $\begin{array}{l}\text { Complex-absent or hypoplastic chamber or valve or common valve: } \\
\text { includes complete atrioventricular septal defect (CAVSD), hypoplastic left heart } \\
\text { syndrome (HLHS), pulmonary atresias, tricuspid atresias, truncus arteriosus, double } \\
\text { inlet left ventricle (DILV), mitral atresia, aortic atresia, congenitally corrected } \\
\text { transposition of the great arteries (CTGA) }\end{array}$ \\
\hline S & $\begin{array}{l}\text { Significant-congenital heart disease requiring operation or intervention } \\
\text { but not included in complex group: includes aortopulmonary window, critical } \\
\text { aortic stenosis, partial AVSD, coarctation, ventricular septal defect (requiring } \\
\text { operation), transposition of the great arteries (TGA), tetralogy of Fallot, total } \\
\text { anomalous pulmonary venous connection (excludes persistent arterial duct, atrial } \\
\text { septal defect) }\end{array}$ \\
\hline M & $\begin{array}{l}\text { Minor-no intervention: } 4 \text { chambers, } 4 \text { valves-includes mainly small ventricular } \\
\text { septal defect (VSD), less severe aortic stenosis, and pulmonary stenosis }\end{array}$ \\
\hline Q & Acquired: includes Kawasaki disease, Marfan's syndrome \\
\hline $\mathrm{O}$ & Normal: used only in conjunction with Down's syndrome \\
\hline $\mathrm{R}$ & Isolated arrhythmia \\
\hline $\mathrm{D}$ & Isolated duct: persistent-not preterm \\
\hline $\mathrm{P}$ & Cardiomyopathies \\
\hline $\mathrm{F}$ & ASD: no operation yet \\
\hline A & ASD: after ASD operation \\
\hline B & Bicuspid aortic valve \\
\hline $\mathrm{N}$ & Isolated arterial duct: preterm \\
\hline $\mathrm{H}$ & Duct requiring operation + minor heart malformation \\
\hline $\mathrm{X}$ & Dextrocardia, where no other abnormality \\
\hline $\mathrm{T}$ & Cardiac tumours \\
\hline
\end{tabular}

NORCAS, 1999.

be picked up in the fetus were demonstrated, using slides and teaching videos. Time for discussion was followed by an afternoon of practical demonstrations on antenatal patients. In the initial teaching we intended to concentrate on the four chambered view, as suggested by Allan and colleagues, and Fermont and associates. ${ }^{12}$ However, the rapid learning curve of most of the ultrasonographers meant that very early on the fetal cardiac examination was extended to include the cardiac outlets. We had incorrectly assumed this to be difficult for an obstetric scanner to include in a routine anomaly scan. Any deviation from the normal appearance or inability to find the four chambers or outlets was considered an indication for referral to the fetal cardiology clinic at Freeman Hospital. A contact telephone number was available at the centre for advice on every working day. The results of referrals were fed back both to clinicians and to ultrasonographers.

Subsequent visits were made by one of us $(\mathrm{AH})$ at six weeks and then again at six and 12 months to identify any problems and attempt to solve them. Individual ultrasonographers were encouraged to visit the regular regional fetal cardiology clinics to perform cardiac scans

Table 2 Prenatal diagnosis of complex/significant congenital heart disease in the northern region

\begin{tabular}{|c|c|c|c|c|c|c|c|c|}
\hline & \multicolumn{2}{|l|}{1994} & \multicolumn{2}{|l|}{1995} & \multicolumn{2}{|l|}{1996} & \multicolumn{2}{|l|}{1997} \\
\hline Birth rate & \multicolumn{2}{|c|}{35026} & \multicolumn{2}{|c|}{$34103(-2.5 \%)$} & \multicolumn{2}{|c|}{$33673(-1.3 \%)$} & \multicolumn{2}{|c|}{$32874(-2.3 \%)$} \\
\hline $\begin{array}{l}\mathrm{C} / \mathrm{S} \text { CHD recognised } \\
\text { prenatally }\end{array}$ & \multicolumn{2}{|c|}{$22(17.6 \%)$} & \multicolumn{2}{|c|}{$33(30.0 \%)$} & \multicolumn{2}{|c|}{$45(36.0 \%)$} & \multicolumn{2}{|c|}{$28(26.9 \%)$} \\
\hline Outcome & TOP & 6 & TOP & 12 & TOP & 23 & TOP & 16 \\
\hline & IUD & 4 & IUD & 1 & IUD & 3 & IUD & 1 \\
\hline & NND & 4 & NND & 7 & NND & 6 & NND & 6 \\
\hline \multicolumn{9}{|l|}{ Registered with NORCAS } \\
\hline Complex & \multicolumn{2}{|l|}{34} & \multicolumn{2}{|l|}{26} & \multicolumn{2}{|l|}{27} & \multicolumn{2}{|l|}{23} \\
\hline Significant & \multicolumn{2}{|l|}{81} & \multicolumn{2}{|l|}{71} & \multicolumn{2}{|l|}{72} & \multicolumn{2}{|l|}{64} \\
\hline Total & \multicolumn{2}{|l|}{115} & \multicolumn{2}{|l|}{97} & \multicolumn{2}{|l|}{99} & \multicolumn{2}{|l|}{87} \\
\hline \multicolumn{9}{|l|}{$\begin{array}{l}\text { Incidence of } \\
\text { CCHD/1000 live }\end{array}$} \\
\hline births & \multicolumn{2}{|l|}{3.3} & \multicolumn{2}{|l|}{2.8} & \multicolumn{2}{|l|}{2.9} & \multicolumn{2}{|l|}{2.6} \\
\hline Number of fetal scans & \multicolumn{2}{|l|}{354} & \multicolumn{2}{|l|}{344} & \multicolumn{2}{|l|}{394} & \multicolumn{2}{|l|}{373} \\
\hline Termination rate & \multicolumn{2}{|l|}{$22.7 \%$} & \multicolumn{2}{|l|}{$36.3 \%$} & \multicolumn{2}{|l|}{$51.1 \%$} & \multicolumn{2}{|l|}{$57 \%$} \\
\hline
\end{tabular}

CCHD, complex or significant congenital heart disease; IUD, intrauterine death; NND, neonatal death; TOP, termination of pregnancy. and to observe. This facility was also available to radiologists, obstetricians, and neonatologists.

\section{DATA COLLECTION}

Since 1985, NORCAS (the Northern Regional Congenital Abnormality Survey) has collected data on congenital abnormalities, as described previously. ${ }^{5}$ This registry now achieves nearly complete ascertainment of all anomalies. Anomalies are notified by sonographers, obstetricians, paediatricians, paediatric cardiologists and pathologists, and information is collected on fetuses, stillborn and live born infants, and from necropsy data. For this study, data were extracted from the survey for positive fetal heart scans and all cases of complex or significant congenital heart disease (CCHD) diagnosed in infancy for 1994, 1995, 1996 and 1997.

The data from 1994 represented the period before training started. The training schedule began at the start of 1995 and was completed in February 1996; 1996 was therefore taken to be the first year in which the effect of training would be assessed.

We used an arbitrary classification of congenital heart disease (table 1) already in use within the northern region and previously published. ${ }^{6}$ Category 1 includes cardiac anomalies or groups of anomalies which are either inoperable, incompletely correctable or only palliatable and classified as complex. Category 2 consists of significant anomalies which if unoperated will carry a high mortality but which can be corrected, leaving the child with a biventricular circulation. Category 3 consisted of minor lesions which do not constitute a threat to life and are considered simple. For the purposes of this study we disregarded this group, as the malformations were less likely to be picked up at routine scanning and would not constitute a reason for intervention in pregnancy.

\section{STATISTICS}

The numbers of cases, outcomes, and recognition rates in 1994 and 1997 were examined using $\chi^{2}$ analysis. A probability value of $\mathrm{p}<0.05$ was considered significant.

\section{Results}

The results are summarised in table 2 .

\section{BIRTH RATE}

At the inception of NORCAS, the birth rate in the northern region was just over 40000 per annum. Reflecting a nation wide trend, the birth rate in the region is falling. Between 1994 and 1995 the fall was $2.6 \%$, between 1995 and 1996, 1.3\%, and between 1996 and 1997, $2.3 \%$.

FETAL SCANS

There was a modest rise in the number of fetal scans/1000 live births over the four years, mainly caused by a rise in referrals because of abnormal cardiac scans recognised by the 
ultrasonographers. The number of "at risk pregnancy" scans is included in these totals and remained static.

CONGENITAL HEART DISEASE UNRECOGNISED BEFORE BIRTH

The number of complex and significant anomalies reported to the registry but unrecognised before birth (total numbers of CCHD

Table 3 Complex/significant congenital heart disease recognised prenatally, followed by termination of pregnancy, 1996

\begin{tabular}{rll}
\hline Patient & Cardiac lesions & Chromosomes \\
\hline 1 & HLHS & $\mathrm{N}$ \\
2 & HLHS & $\mathrm{N}$ \\
3 & HLHS & $\mathrm{N}$ \\
4 & HLHS & $\mathrm{N}$ \\
5 & HLHS & $\mathrm{N}$ \\
6 & HLHS-EFE, tiny LV & $\mathrm{N}$ \\
7 & LV hypoplasia, aortic hypoplasia, LVH+ & $\mathrm{N}$ \\
8 & Critical aortic stenosis-very small LV, EFE & $\mathrm{N}$ \\
9 & Hypoplastic LV, DORV, mitral atresia & $\mathrm{N}$ \\
10 & Tetralogy of Fallot, small LV, pulmonary atresia, & \\
& cleft lip and palate & $\mathrm{N}$ \\
11 & Multiple VSDs, cleft lip and palate & Translocation, chromosome 10 \\
12 & Severe Ebstein's anomaly & $\mathrm{N}$ \\
13 & TGA, pulmonary atresia, renal agenesis & Trisomy 18 \\
14 & Large confluent VSD, severe aortic hypoplasia, & \\
& tetralogy of Fallot & Trisomy 21 \\
15 & Perimembranous VSD, pulmonary atresia & Trisomy 21 \\
16 & CAVSD, hypoplastic LV & Trisomy 21 \\
17 & CAVSD, hypoplastic LV, DORV & Partial deletion, chromosome 6 \\
18 & CAVSD, hypoplastic LV & $\mathrm{N}$ \\
19 & CAVSD, hypoplastic LV, TGA & $\mathrm{N}$ \\
20 & CAVSD, pulmonary atresia & $\mathrm{N}$ \\
21 & CAVSD, TGA & $\mathrm{N}$ \\
22 & CAVSD, LVOTO, renal agenesis & Trisomy 21 \\
23 & PAVSD, hypoplastic LV & \\
& &
\end{tabular}

Total: five major and two minor chromosomal problems, two lethal non-cardiac abnormalities, three non-cardiac surgical abnormalities.

CAVSD, complete atrioventricular septal defect; DORV, double outlet right ventricle; EFE, endocardial fibroelastosis; HLHS, hypoplastic left heart syndrome; LV, left ventricle; LVH, left ventricular hypertrophy; LVOTO, left ventricular outflow tract obstruction; N, normal; PAVSD, partial atrioventricular septal defect; TGA, transposition of the great arteries.

Table 4 Complex/significant congenital heart disease recognised but fetus not aborted, 1996

\begin{tabular}{|c|c|c|c|}
\hline & Patient & Cardiac lesions & Chromosomes \\
\hline \multirow[t]{3}{*}{ IUD } & 1 & $\begin{array}{l}\text { Inlet VSD, straddling tricuspid valve complete } \\
\text { heart block }\end{array}$ & Trisomy 18 \\
\hline & 2 & CAVSD, hydrops, bradycardia, left atrial isomerism & $\mathrm{N}$ \\
\hline & 3 & Common arterial trunk type 1 & $\mathrm{~N}$ \\
\hline \multirow[t]{6}{*}{ NND } & 1 & Double chambered RV, RA++, TR++ & $\mathrm{N}$ \\
\hline & 2 & CAVSD, hypoplastic LV, coarctation, coartectomy & Trisomy 21 \\
\hline & 3 & Tetralogy of Fallot, diaphragmatic hernia & $\mathrm{N}$ \\
\hline & 4 & Tetralogy of Fallot, DORV, polycystic kidneys & $\mathrm{N}$ \\
\hline & 5 & $\begin{array}{l}\text { TGA, DORV, interrupted aortic arch, tetralogy } \\
\text { of Fallot }\end{array}$ & $\mathrm{N}$ \\
\hline & 6 & Coarctation, RV and RA enlargement & Trisomy 13 \\
\hline \multirow[t]{13}{*}{ Alive } & 1 & Confluent VSD, LVOTO, complete heart block & $\mathrm{N}$ \\
\hline & 2 & $\begin{array}{l}\text { Multiple myocardial plaques and ventricular } \\
\text { dysfunction }\end{array}$ & $\mathrm{N}$ \\
\hline & 3 & CAVSD, heart in right chest & $\mathrm{N}$ \\
\hline & 4 & Tetralogy of Fallot, exomphalos & $\mathrm{N}$ \\
\hline & 5 & Perimembranous VSD, coarctation & $\mathrm{N}$ \\
\hline & 6 & $\begin{array}{l}\text { Williams syndrome, pulmonary valve stenosis, } \\
\text { exomphalos }\end{array}$ & $\mathrm{N}$ \\
\hline & 7 & Large confluent perimembranous VSD & $\mathrm{N}$ \\
\hline & 8 & $\begin{array}{l}\text { CAVSD, tetralogy of Fallot, exomphalos } \\
\text { Goldenhaar's syndrome }\end{array}$ & $\mathrm{N}$ \\
\hline & 9 & Rhabdomyoma, single large & $\mathrm{N}$ \\
\hline & 10 & TGA & $\mathrm{N}$ \\
\hline & 11 & Coarctation, $\mathrm{R}$ heart enlargement & $\mathrm{N}$ \\
\hline & 12 & $\begin{array}{l}\text { DIRV, straddling left AV valve, hypoplastic LV, } \\
\text { DORV }\end{array}$ & $\mathrm{N}$ \\
\hline & 13 & Multiple rhabdomyomata, SVT, tuberous sclerosis & $\mathrm{N}$ \\
\hline
\end{tabular}

Total, 22 cases: three serious chromosomal abnormalities; 13 survivors.

$\mathrm{AV}$, atrioventricular; CAVSD, complete atrioventricular septal defect; DIRV, double inlet right ventricle; DORV, double outlet right ventricle; IUD, intrauterine death; LV, left ventricle; NND, neonatal death; LVOTO, left ventricular outflow tract obstruction; $\mathrm{N}$, normal; R, right; RA, right atrium; RV, right ventricle; SVT, supraventricular tachycardia; TGA, transposition of the great arteries; TR, tricuspid regurgitation; VSD ventricular septal defect. registered with NORCAS minus antenatally detected cases) fell from 103 in the first year to 76 in the fourth year ( $p=0.076, \mathrm{NS})$. The breakdown into "complex" and "significant" in table 2 suggests that although both groups fell, the reduction in the complex group was greater, though this did not reach significance.

PRENATAL POSITIVE SCANS

In the pretraining year only 22 serious anomalies were picked up by the obstetric units. Six of these fetuses were aborted and eight died either in utero or in the neonatal period, confirming the serious nature of the lesions. In the intermediate year, the pick up rate increased to 33, of whom 12 were aborted and another eight died in utero or in the neonatal period. In year 3 , the pick up of serious lesions rose steeply to a total of 45 . Of these 23 were aborted and another nine died in utero or in the neonatal period (tables 3 and 4 show the anatomical findings in the CCHD group in 1996, whether terminated or not). In year 4 , only 28 serious anomalies were picked up antenatally. Of these 16 were aborted, one died in utero, and six died in the neonatal period. The overall change in pick up rate between 1994 and 1997 was significant $(\mathrm{p}=0.033)$.

Thus immediately following the training period the detection rate of CCHD at 18-20 weeks' gestation more than doubled, from $17.6 \%$ to $36 \%$, and then fell to $26.9 \%$ in the fourth year. Over the four years the termination rate rose from $22.7 \%$ to $57.0 \%(\mathrm{p}=0.035)$.

\section{INCIDENCE OF SIGNIFICANT CONGENITAL HEART} DISEASE

The liveborn incidence of complex and significant congenital heart disease fell between 1994 and 1995, showed no change between 1995 and 1996, and then fell again in 1997. The fall in incidence between 1994 and 1997 is highly significant $(\mathrm{p}<0.0001)$.

\section{Discussion}

Fetal cardiac scanning in our region was a natural progression from paediatric echocardiography. Ultrasound machines already existed in the paediatric cardiology department and almost all the district general hospitals. The obstetric population enjoyed a scan rate in excess of $96 \%{ }^{4}$ and the NORCAS facility provided an ideal assessment tool. The results of this unique audit show that a training programme in fetal echocardiography for obstetric ultrasonographers with access to a regional centre can increase the detection rate of CCHD at 18-20 weeks' gestation.

Initially most fetal scans were carried out on "at risk" pregnancies because of a positive family history or maternal disease such as diabetes, or because a non-cardiac malformation or abnormality known to be associated with congenital heart disease had been found. The offspring study of Burn and colleagues suggested that siblings of affected children had a $2 \%$ recurrence risk, children whose fathers had congenital heart disease also had a $2 \%$ recurrence risk, and children of mothers with congenital heart disease had a $5 \%$ risk or 
greater. ${ }^{7}$ However, the pick up rate in scanning such pregnancies is unlikely to be high (according to the Guy's Hospital experience, less than $10 \%^{8}$ ) and would have a small effect on the prevalence of congenital heart disease.

High resolution echocardiography with Doppler can identify most forms of serious congenital heart disease from 17-18 weeks' gestation if the scan is carried out by a skilled echocardiographer. Even if there were enough paediatric echocardiographers to carry out all these scans, it has been estimated that each would have to scan for 300 hours to pick up one serious congenital heart lesion. ${ }^{2}$ We took the pragmatic approach and encouraged ultrasonographers to try to obtain the views of the four cardiac chambers and the cardiac outflows during routine anomaly scans, having confirmed that this produced the most effective referrals. There was a gradual increase in referrals with abnormal cardiac views, and ultrasonographers quickly learned to recognise the cardiac outlets. However, the improvement was patchy and encouraged us to proceed with a prospective training programme involving all maternity units throughout the region.

There was widespread though not universal enthusiasm for the project. Some units were concerned that their ultrasound machines were too old and unsophisticated to allow them to participate. One centre with a high level of enthusiasm had a low pick up rate to begin with, which improved dramatically after a new ultrasound system was installed. There was also concern that the cardiac scan would add significantly to the scanning time. This has not proved to be a problem.

The teaching programme was tailored to be carried out by a consultant paediatric cardiologist and an echo technician working in paediatric cardiology. The training schedule was arranged to allow both on site teaching and off site day visits to the regional centre. It was intended to complete the schedule, detailed in Methods, within the second year. In the event all the units were covered by the end of February 1996.

Our results show that a simple programme of teaching without major financial implications produced a significant change in the clinical diagnostic expertise of ultrasonographers in the northern region maternity units. The increased success in picking up congenital heart disease engendered enthusiasm for the technique and provided a better service, which the obstetricians and the parents greatly appreciate. The concern shown when consultant obstetricians discovered the absence of the fetal cardiologist on holiday demonstrates the degree to which they now depend on the service.

We are not, however, entirely satisfied with the results of our training programme. If we did recognise $36 \%$ of serious abnormalities in 1996 , we should not forget that $64 \%$ came to delivery undiagnosed. Why did we miss them? Is it correct to say that as the expertise of the ultrasonographers improves we should in time be able to identify most connection anomalies (that is, the serious ones) before birth ${ }^{8}$
Furthermore, why did the detection rate fall to $26.9 \%$ in 1997 ? There is, as we have already stated, the problem of inherent variability in dealing with relatively small numbers. In addition, as has been demonstrated by Wren and colleagues, ${ }^{6}$ the incidence of congenital anomalies varies quite widely from year to year. It may be that in 1997 the initial effects of the training programme were beginning to diminish. However, early results from 1998 suggest that the pick up rate is similar to 1996 and that the termination rate has risen again. More time is required with further data collection to confirm the trends which this study suggests.

Our findings are given added weight by a recent report on behalf of the British Paediatric Cardiac Association. ${ }^{9}$ This collaborative study looked at fetuses with heart lesions diagnosed between 1993 and 1995 in 17 paediatric cardiac centres in the UK. The overall instance of CCHD is in line with the levels in our series. Detection rates varied quite considerably, with levels well above the national average clustered in the south east of England, probably because of proximity to and contact with the Guy's Hospital fetal medicine department. This is the longest standing fetal cardiology unit in the country and has provided a teaching programme for many years in the south east. This observation supports our finding that detection can be significantly increased by appropriate teaching programmes.

Although fetal cardiology has added to our knowledge of congenital heart disease in fetal life, its principal function is to identify malformations which are associated with a poor or hopeless outlook so that parents have the option of terminating the pregnancy. The recurrence rate of congenital heart disease is not high. This makes the option of termination acceptable to the majority of parents. It is interesting that the proportion of parents choosing termination rose by so much. The figure of $57 \%$ is similar to that quoted in a very large study from Guy's Hospital. ${ }^{8}$ For the most severe malformations, such as hypoplastic left heart syndrome, the rate was close to $90 \%$. The increase in termination of pregnancies is difficult to explain, as the counselling team has remained constant. However, malformations are now referred more promptly and earlier than previously, giving parents more time. It is interesting that the termination rate in Bull's national study seems to show a decline while the northern region rate continues to rise. ${ }^{9}$ Without exception, termination was requested only for lethal malformations or a combination of abnormalities without the future option of correction. While the presence of serious noncardiac malformations and chromosomal abnormalities played a part in the decision to terminate the pregnancy, we have not specifically addressed this aspect.

A simple teaching programme carried out by only two health care professionals has produced an increase in the rate of detection of CCHD at 18-20 weeks' gestation. The benefit to the parents of reliable early prenatal diagnosis and the possibility of changing the outcome is obvious and widely appreciated. It is impos- 
sible in this particular study to weigh the benefits to the parents and families of in utero cardiac diagnosis against the financial and staffing implications for health care providers. It is interesting, however, to consider that the most severe cardiac lesions - such as hypoplasia of the right or left heart-lead increasingly, if the baby survives pregnancy, to multiple surgical events and prolonged hospital treatment, resulting in considerable stress to the family as well as major financial implications for the regional paediatric cardiology centre.

If the promise of this initial programme is fulfilled and sustained in the former northern region over subsequent years then there may be a significant change in the spectrum of serious congenital heart disease. If our results can be further enhanced by modifications to the training schedule and the initiative extended to other regions, then the effect on congenital heart disease nationally might be significant.
1 Allan LD, Crawford DC, Chita SK, et al. Prenatal screening for congenital heart disease. BMF 1986;292:1717-19.

2 Fermont L, De Geeter B, Aubry J, et al. A close collaboration between obstetricians and paediatric cardiologists allows antenatal detection of severe congenital malformations by $2 \mathrm{D}$ echocardiography. In: Doyle EF, Engle ME, Gersony WM, et al, eds. Pediatric cardiology: proceedings of the Second World Congress. New York: Springer Verlag, 1986:34-7.

3 Sharland GK, Allan LD. Screening for congenital heart disease prenatally: results of a $2 \frac{1}{2}$ year study in South East Thames region. Br $\mathcal{F}$ Obstet Gynaecol 1992;99:220-5.

4 Wyllie JP, Wren C, Hunter S. Screening for cardiac malformations. Br Heart f 1994;71 (suppl):20-7.

5 Renwick $M$. The regional fetal abnormality survey: an audit of its recognition and management. Arch Dis Child 1992;67:770-4.

6 Wren C, Richmond S, Donaldson L. Temporal variability in birth prevalence of cardiovascular malformations. Heart 2000;83:414-19.

7 Burn J, Brennan P, Little J, et al. Recurrence risks in offspring of adults with major heart defects: results from first cohort of British collaborative study. Lancet 1998;351: 311-16.

8 Allan LD, Sharland GK, Anderson RH, et al. Prospective diagnosis of 1006 consecutive cases of congenital heart disease in the fetus. $7 \mathrm{Am}$ Coll Cardiol 1994;23:1452-8.

9 Bull C. Current and potential impact of fetal diagnosis on prevalence and spectrum of serious congenital heart disease at term in the UK. Lancet 1999;351:1242-7.

\section{Treatment of tachyarrhythmia induced syncopes by angioplasty of the right coronary artery}

Recurrent syncopes in a 68 year old patient prompted further examination in addition to a neurological check. Holter monitoring was performed without antiarrhythmic medication. The ECG showed accelerated polymorphic ventricular tachycardias with degeneration to ventricular flutter and spontaneous termination (left). The tachycardias were documented twice and were sustained for up to two minutes. Coronary angiography revealed $90-95 \%$ proximal stenosis of the right coronary artery with an intraluminal filling defect (right). The following day a successful angioplasty of the affected vessel, with implantation of an intracoronary stent $(3.5 \mathrm{~mm})$, was performed. No more arrhythmic episodes were documented afterwards. Even the programmed stimulation of the right ventricle was inconspicuous and no further ventricular tachycardias were inducible.

Ventricular tachyarrhythmias in ischaemic heart disease indicate electrical instability of the affected myocardium. Because of poor myocardial tissue perfusion, the conduction system is influenced by modulation of the membrane potential, changes in the refractory time, and appearance of conduction blocks followed by re-entry mechanisms. Thus, high degree ventricular arrhythmias can occur in the absence of a defined electrophysiological substrate such as a myocardial scar.

The question of whether revascularisation such as angioplasty or surgical revascularisation can restore the electrical stability remains open. It has been shown that patients with a normal left ventricular function and no inducible ventricular tachycardias at electrophysiological examination after revascularisation benefit from revascularisation.

In our case we assume the polymorphic ventricular tachycardias were successfully treated by angioplasty. This case underlines the necessity of performing a cardiological as well as a neurological examination in patients with recurrent syncopes.

H REITER G HASENFUSS C UNTERBERG

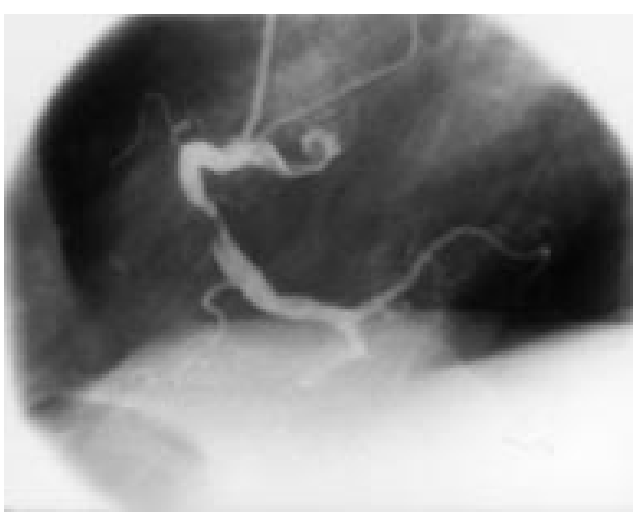

